\title{
Energy Harvesting Powered Wireless Sensor Nodes With Energy Efficient Network Joining Strategies
}

\author{
Zheng Jun Chew, Tingwen Ruan and Meiling Zhu \\ College of Engineering, Mathematics and Physical Sciences \\ University of Exeter \\ EX4 4QF Exeter, United Kingdom \\ z.j.chew@exeter.ac.uk; tr287@exeter.ac.uk;m.zhu@exeter.ac.uk
}

\begin{abstract}
This paper presents strategies for batteryless energy harvesting powered wireless sensor nodes based on IEEE 802.15.4e standard to join the network successfully with minimal attempts, which minimizes energy wastage. This includes using a well-sized capacitor and different duty cycles for the network joining. Experimental results showed a wireless sensor node that uses a $100 \mathrm{mF}$ energy storage capacitor can usually join the network in one attempt but multiple attempts may be needed if it uses smaller capacitances especially when the harvested power is low. With a duty-cycled network joining, the time required to form a network is shorter, which reduces the overall energy usage of the nodes in joining the network. An energy harvesting powered wireless sensor network (WSN) was successfully formed in one attempt by using the proposed methods.
\end{abstract}

Keywords-batteryless, energy harvesting, industrial, SmartMesh IP, wireless sensor network.

\section{INTRODUCTION}

Wireless sensor nodes are increasingly popular for industrial applications especially with the rise of the industrial Internet of Things (IIoT) since they can be easily installed over a wide area or without much disruption on existing assets in a more cost effective manner than their wired counterparts [1]. However, batteries that are commonly used to power wireless devices have limited capacity and are often one of the causes of node failure once depleted [1]. Regular battery replacement is required to keep the nodes working, which is costly and polluting if the used batteries are not properly disposed [2].

Energy harvesting has emerged to be a potential solution to supply energy to WSNs for perpetual operation [2]. Energy harvesting converts energy sources such as light [3], fluid flow [4], vibration [5], and heat [6] from the ambient environment into electrical energy to power the wireless sensor nodes. The types of energy sources available for harvesting vary with time and locations [7], which means the harvested energy levels may be different from node to node [8]. Therefore, in many cases, energy harvesting powered wireless sensor become active to send data to the base station only when energy is available [9], [10]. This may not suit most industrial applications, which prefer a predictable behavior [1].

One potential solution is to form a well-managed WSN based on the Time Synchronized Channel Hopping (TSCH) technique. TSCH is compliant to the industrial IEEE $802.15 .4 \mathrm{e}$ standard [11]. A TSCH based WSN may consists of up to 100

This work is supported by EPSRC grant through En-ComE project (EP/K020331/1). nodes and a network manager to control the schedule of the wireless communication among the nodes with a reliability of over $99.999 \%$ while consumes about $80 \%$ less power than standard IEEE 802.15.4 networks for its communication [14]. Therefore, it is possible to use energy harvesting to power TSCH based WSNs due to their low power requirement.

However, the network join process, which is the first process that the nodes have to complete to form a network is very power hungry. So far, many studies on TSCH-based networks are battery powered [11]-[13]. Attempts to power TSCH nodes using energy harvesting were either unsuccessful [14] or took 12 hours to charge up a $0.33 \mathrm{~F}$ capacitor for only $12 \mathrm{~s}$ of initial startup [15]. Thus, there is a need to address the network join issues of energy harvesting powered wireless sensor nodes so that the nodes can truly benefit from the abundance of energy in the environments for perpetual operation of WSNs.

This paper presents strategies to enable energy harvesting powered wireless sensor nodes to join a network successfully with minimal attempts by incorporating a duty-cycled network join process with an appropriate capacitor size. This reduces the number of times of the network join process that the nodes have to go through, which saves energy especially in low level energy harvesting where the limited energy is unlikely to allow the nodes to keep trying to join the network. A WSN was formed by using SmartMesh IP chips, which are a commercially available platform based on TSCH that have been widely deployed [16]. Energy harvesting from airflow and vibration were each used to power one node that adopts the proposed approaches.

\section{PRoposed Methods}

\section{A. Network Joining Duty Cycle}

Network joining of wireless sensor nodes involves listening to the advertisement from the network manager, sending a join request, and waiting for a reply from the network manager before the nodes are finally connected to a network. The time spent by the nodes in any of the three stages of the network joining process is random depending on the structure and characteristic of the network. The network manager can only reply to one node at a time [17]. Therefore, some nodes may stay in one of the three stages until their energy is used up and have to wait until they have enough energy again, especially when there are two or more nodes are trying to join the network simultaneously. 
By configuring the network joining process of the nodes to be periodical where the nodes are active or sleeping based on the duty cycle set, there will be time for two things. First, the capacitor can be recharged if there is energy to be harvested when the radio is off. This can keep the nodes powered on for longer with a better chance of joining the network. Secondly, the network manager can communicate with other nodes when the node that was initially attempting to join the network went to sleep. Other nodes that have been powered up would be able to be connected to the network instead of having to wait until the first node has completed its connection in the case of network joining without duty cycle. Energy can be saved as less time is spent on waiting for one node to end its operation.

\section{B. Capacitor Size}

Capacitors are generally used to store energy in batteryless systems. A system with an appropriate capacitance $C$ can be selected once the time $\mathrm{d} t$ for the capacitor to keep the nodes powered on has been set, the operating voltage range of the system and the average current $I$ consumed by the nodes during the network join process are known, by using (1).

$$
C=I \frac{\mathrm{d} t}{V_{\max }-V_{\min }}
$$

where $V_{\max }$ and $V_{\min }$ are the maximum and minimum operating voltage range, respectively.

Equation (1) can also be used to determine the time to charge up a given $C$ from $V_{\min }$ to $V_{\max }$ if the current that flows into the capacitor is known. Since it is desirable to charge up a capacitor of the energy harvesting system as soon as possible for the nodes to start, the capacitance chosen should consider a balance between the discharge and recharge time based on the outward and inward current of the capacitor, respectively. For example, if the output power from the energy harvester is high, the current after power conditioning could be sufficiently high to provide the nodes with the amount they required for the network joining or quickly recharged the capacitor if the first network join attempt failed. Thus, smaller capacitances can be used.

\section{IMPLEMENTATION AND TEST}

The WSN in this work is composed of a DC9001B network manager and two DC9003A-B nodes that have the SmartMesh IP LTC5800 chip [16]. The network manager was connected to a computer to get a steady power supply so that it can do the scheduling task without interruption. The nodes were each powered by energy harvested from vibration and airflow, where both sources are abundant in industrial environments.

\section{A. System Design}

Fig. 1 shows the energy harvesting powered node that consists of an energy harvester, an optional rectifier for $\mathrm{AC}$ input, a power management circuit (PMC), an energy storage device, an energy-aware interface (EAI), and a wireless sensor node [9], [10]. The rectifier converts AC voltage from a M8528P2 macro-fiber composite (MFC) [18], which is bonded on a carbon fiber composite as the vibration energy harvester (VEH) into DC. The airflow energy harvester (AEH) consists of a turbine and a DC generator [4], which does not require rectification. The PMC converts the voltage from the energy harvesters to up to $3.3 \mathrm{~V}$ to charge up the energy storage device, which is a capacitor. The PMC has a maximum power point controller to enable maximum power transfer from the energy harvesters to the capacitor [19]. For the VEH, which usually generates high voltage, a buck topology is used [9], [10], [19], while for the AEH, which usually has low output voltage [4], a boost topology is used in the PMC. The EAI keeps the wireless sensor node disconnected from the capacitor so that energy can be accumulated [20]. Once the capacitor voltage reaches $3.15 \mathrm{~V}$, the EAI connects the node to the capacitor, which subsequently turns on the node [21]. The node stays active until the voltage drops to $2.25 \mathrm{~V}$, and then the EAI disconnects the node from the capacitor again to allow the capacitor to be recharged to $3.15 \mathrm{~V}$.

\section{B. Testing Methods}

The first test was to study the effect of duty-cycle on the network joining. The two nodes were set to join the network at the same time with the same duty cycle of $100 \%$ first, and then with $50 \%$. The duty cycle was configured by setting the 'joindc' parameter of the LTC5800 chip as 128 for $50 \%$ and 255 for $100 \%$ [22]. The time required by the nodes to be connected to the network successfully at different duty cycles were compared. The nodes were powered by the power supply since the time required by the nodes to join the network could be very long where energy harvesting might not be able to sustain it. 50 measurements were taken from each node in each test to determine the probability of joining the network successfully.

Then, different capacitances were used in this experiment to determine their effect on the network joining. Only the VEH was used to power one node because it is easier to simulate low power conditions using the MFC that usually has high output voltage above the minimum operating voltage of the buck converter [23]. Peak-to-peak strain levels of $300 \mu \varepsilon$ at $2 \mathrm{~Hz}$ and $600 \mu \varepsilon$ at $10 \mathrm{~Hz}$ were applied onto the VEH using an Instron E10000 machine. The strain and frequency range used is typical for structures such as bridges [24], frame structures [25], and aircrafts [26]. The number of attempts and time required by the wireless sensor node to join the network in each of the tested conditions were measured. Up to 4 attempts will be recorded in each scenario before the measurement is stopped as a node is considered lost by the network manager after 5 retries [22].

Finally, an energy harvesting powered WSN was formed by two nodes that were each powered by AEH and VEH. Different network join duty cycles and capacitors were used to study their effects on the network joining process. The Instron E-10000 machine was set to apply a peak-to-peak strain level of $600 \mu \varepsilon$ at $10 \mathrm{~Hz}$ onto the VEH. A wind generator with tunable airflow speed was used to generate airflow towards the AEH. The output power from the AEH was varied to charge up the capacitor of the node at different rates according to the intended tests.

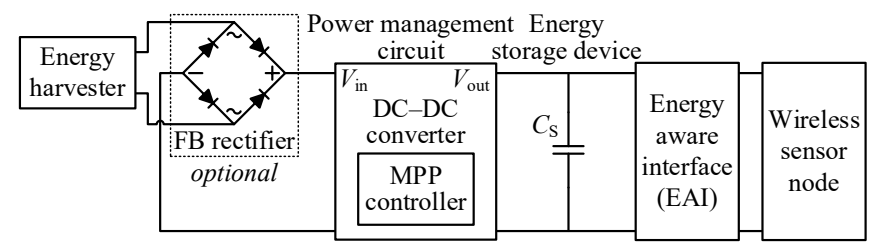

Fig. 1. Block diagram of the energy harvesting powered node. 


\section{RESULTS AND DISCUSSION}

\section{A. Network Join Duty Cycle}

Fig. 2 shows the distribution of the nodes that join the network successfully within certain timeframe without considering the sleep time. The sleep current is very low if compared with other processes. Therefore, the sleep time can be disregarded here since it can be considered negligible in terms of energy usage. If the sleep time was considered, the network join time using the duty cycle of $50 \%$ would almost double.

When two nodes begin to join the network at the same time at $100 \%$ duty cycle, the occurrences of joining the network successfully in $6 \mathrm{~s}$ is relatively high, but it could also take more than $30 \mathrm{~s}$. When the duty cycle is reduced to $50 \%$, the energy intensive process during the network join reduces to $27 \mathrm{~s}$. Still using the duty cycle of $50 \%$ but with one of the nodes begin the network join later by $2 \mathrm{~s}$, a greater improvement was achieved. The energy intensive process reduces to a minimum of $2 \mathrm{~s}$ to less than $22 \mathrm{~s}$. This indicates the gap time of a node with a dutycycled network joining process allows the network manager to communicate with the other nodes, which reduces the waiting time and increases the chance of the other nodes to be connected to the network sooner while they still have the energy.

In all 3 cases, the number of occurrences that the nodes joined the network successfully within $15 \mathrm{~s}$ is relatively high. Therefore, a capacitor that can supply energy to the nodes for at least $15 \mathrm{~s}$ during the network joining process is preferable.
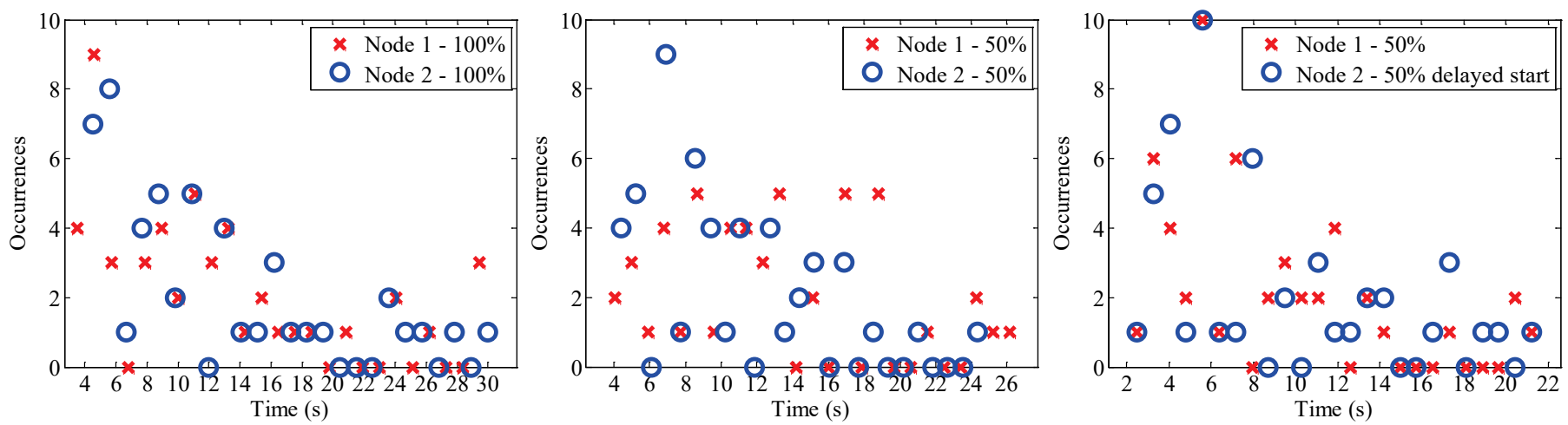

Fig. 2. Occurrences of successful network join of the nodes within certain timeframes at different test conditions. The markers are the midpoints of the bins in the time distribution graphs.

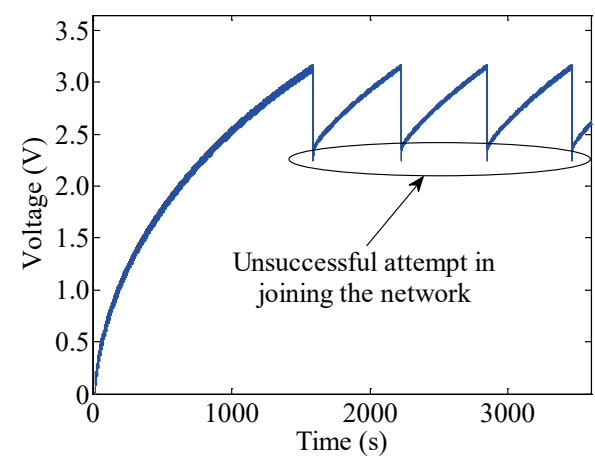

(a)

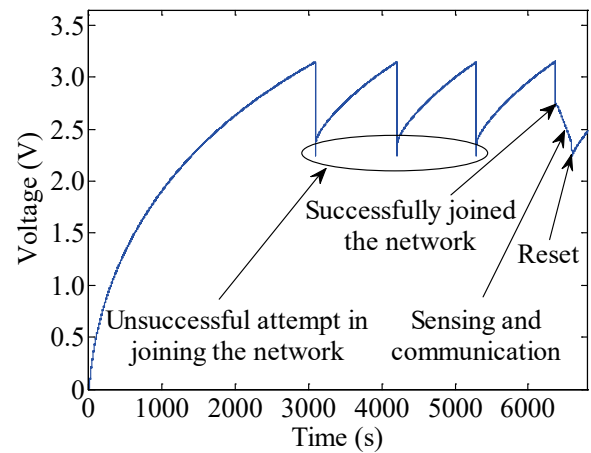

(b)

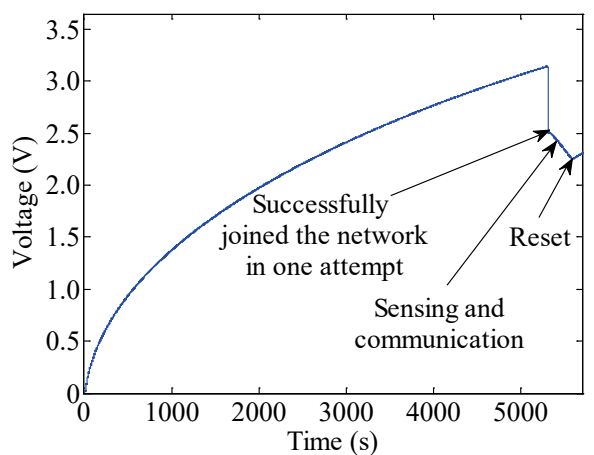

(c)

Fig. 3. Voltage measured across the energy storage capacitor when a peak-to-peak stain loading of $300 \mu \varepsilon$ at $2 \mathrm{~Hz}$ was applied onto the VEH using a supercapacitor of: (a) $33 \mathrm{mF}$, (b) $50 \mathrm{mF}$, and (c) $100 \mathrm{mF}$.

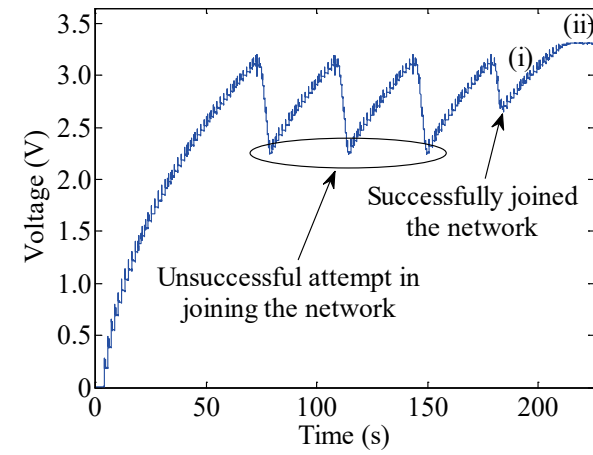

(a)

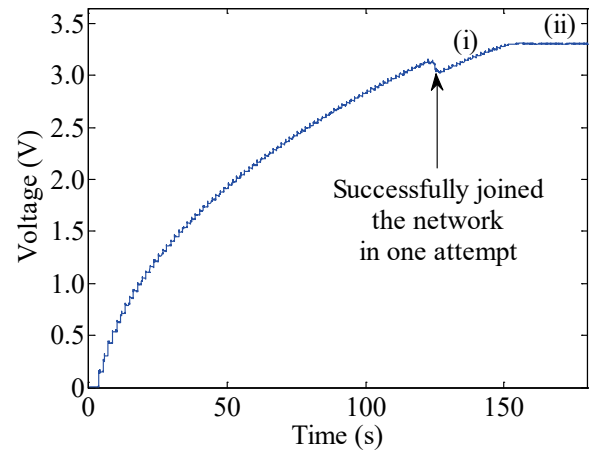

(b)

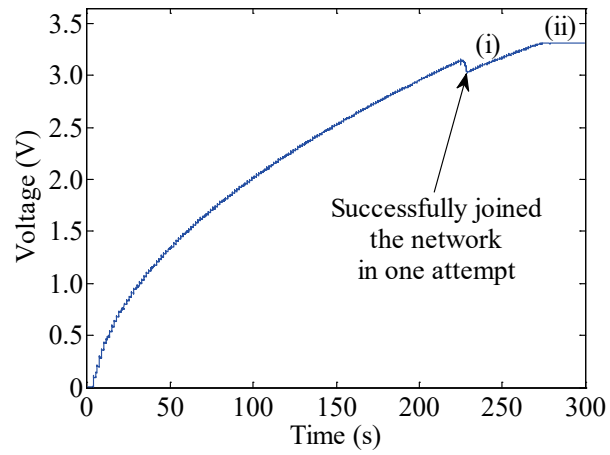

(c)

Fig. 4. Voltage measured across the energy storage capacitor when a peak-to-peak stain loading of $600 \mu \varepsilon$ at $10 \mathrm{~Hz}$ was applied onto the VEH using a supercapacitor of: (a) $33 \mathrm{mF}$, (b) $50 \mathrm{mF}$, and (c) $100 \mathrm{mF}$. 


\section{B. Capacitor Values}

The most power hungry process during the network join process of a TSCH node is the listening process, consuming around $5.5 \mathrm{~mA}$ of current [15]. $C$ of $92 \mathrm{mF}$ is obtained by substituting the values of $15 \mathrm{~s}, 5.5 \mathrm{~mA}, 3.15 \mathrm{~V}$, and $2.25 \mathrm{~V}$ into $\mathrm{d} t, I, V_{\max }$, and $V_{\min }$ in (1), respectively. Therefore, a $100 \mathrm{mF}$ capacitor was chosen after some possible tolerances is taken into account. For comparison purposes, two other capacitors of 33 $\mathrm{mF}$ and $50 \mathrm{mF}$ were also used. Since there was only one node, a join duty cycle of $100 \%$ was used.

In the first test where a peak-to-peak strain level of $300 \mu \varepsilon$ at $2 \mathrm{~Hz}$ was applied onto the VEH, the wireless sensor node could not join the network in all the four attempts when the capacitor of $33 \mathrm{mF}$ was used as shown in Fig. 3(a). This is because that capacitor can only supply energy for a short period of time to the node to join the network. However, the node joined the network successfully in the fourth attempt using the $50 \mathrm{mF}$ capacitor and in one attempt using the $100 \mathrm{mF}$ capacitor, as shown in Figs. 3(b) and (c), respectively. Once the nodes are connected to the network, the capacitor voltage drops at a slower rate than in the network joining process until it reaches $2.25 \mathrm{~V}$. This is because the output power of the power management circuit is $86.7 \mu \mathrm{W}$ when the VEH was excited by a peak-to-peak strain loading of $300 \mu \varepsilon$ at $2 \mathrm{~Hz}$. This power is less than the minimum requirement for sensing and transmission of about $200 \mu \mathrm{W}$ [11]. The nodes were then disconnected from the capacitor by the EAI to allow the energy to be accumulated in the capacitor.

When a higher peak-to-peak strain level of $600 \mu \varepsilon$ at $10 \mathrm{~Hz}$ was applied onto the $\mathrm{VEH}$, the output power from the power management circuit is $2.53 \mathrm{~mW}$. This time, the node managed to join the network successfully with all the different capacitors used. Fig. 6(a) shows it still requires several attempts using the $33 \mathrm{mF}$ capacitor. This agrees with the previous test that a smaller capacitance holds less energy that can only power the nodes for a short network join process, which usually has a lower chance of connecting to the network successfully. However, in this case, the energy used by the nodes comes from the capacitor and the higher power from the VEH. This increases the time of powering the node and the chance of joining the network successfully than the test that uses the lower strain level and frequency. Results in Figs. 6(b) and 6(c) show the wireless sensor node can join the network successfully in one attempt using the 50 and $100 \mathrm{mF}$ capacitors, respectively. The larger capacitors can store more energy to allow the nodes to stay in the network joining process for a longer time, which increases the chance of joining in one attempt. Once the wireless sensor nodes have joined the network successfully, they proceed to do the sensing and communication tasks. This time the energy is enough for the operations of the nodes with surplus energy to charge the capacitors at the same time as indicated by (i) in all three cases. The capacitors were eventually charged to $3.3 \mathrm{~V}$ as indicated by (ii) while the node continues to operate. As the power from the energy harvester is usually too low to sustain the network joining of the node directly or quickly recharge a capacitor for another attempts, the $100 \mathrm{mF}$ capacitor that can provide energy to the node for a time that is usually long enough to join the network in one attempt is a reasonable choice.

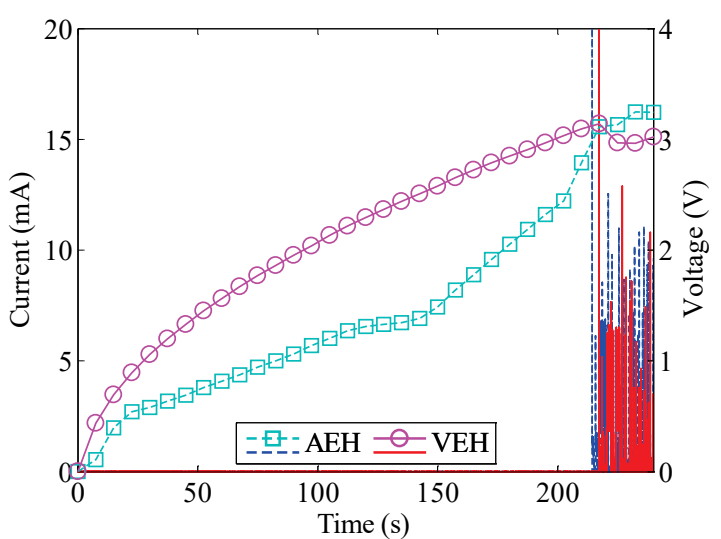

(a)

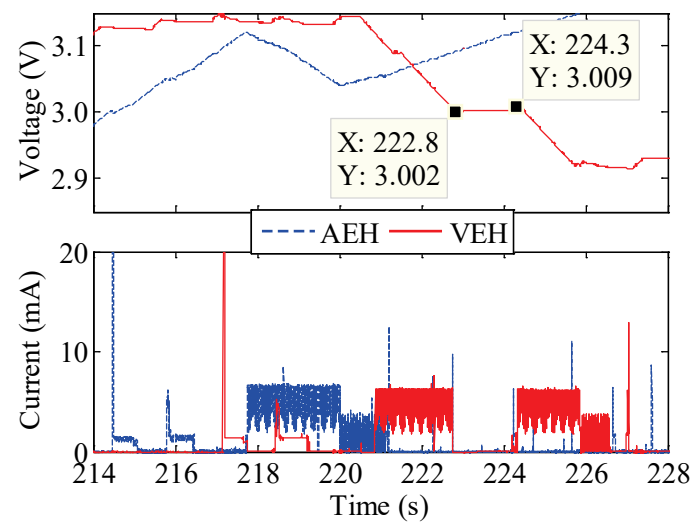

(b)

Fig. 5. (a) Measured voltage across the energy storage capacitor and current consumption of the wireless sensor nodes powered by AEH and VEH using a capacitor size of $100 \mathrm{mF}$ and network join duty cycle of $50 \%$. (b) Enlarged view of the measurements when both nodes successfully joined the network.

TABLE I. Network JoIn StATUS OF THE ENERgy HARVESTING Wireless SENSOR NODES USING DifFERENT TEST CONDITIONS

\begin{tabular}{|c|c|c|c|c|}
\hline \multirow[b]{2}{*}{ Test } & \multirow{2}{*}{$\begin{array}{c}\text { Capacitor } \\
(\mathrm{mF})\end{array}$} & \multirow{2}{*}{$\begin{array}{l}\text { Join duty } \\
\text { cycle (\%) }\end{array}$} & \multicolumn{2}{|c|}{ Network join status } \\
\hline & & & $\begin{array}{c}\text { VEH Powered } \\
\text { Node }\end{array}$ & $\begin{array}{c}\text { AEH Powered } \\
\text { Node }\end{array}$ \\
\hline 1 & 100 & 100 & Failed & Failed \\
\hline 2 & 22 & 50 & Successful & Failed \\
\hline 3 & 100 & 50 & Successful & Successful \\
\hline
\end{tabular}

\section{Energy Harvesting Powered WSN}

Finally, based on these findings, the two energy harvesting powered nodes were designed with $100 \mathrm{mF}$ capacitors and a join duty cycle of $50 \%$. Fig. 5 shows that the nodes can join the network in a relatively short time with just one attempt. An enlarged view of the measurements shows that the capacitor is recharged when the nodes go to sleep. This shows a duty-cycled network join process indeed allows the energy storage device to be replenished when the nodes go to sleep. For comparison purposes, other test conditions were also used as summarized in Table I. It can be seen that the nodes can quickly form the network using the proposed methods while they failed in other conditions. 


\section{CONCLUSION}

A batteryless WSN based on the IEEE 802.15.4e standard has been presented. Two different energy harvesters namely, airflow and vibration were each used to power up a wireless sensor node to form the WSN using the proposed approaches of duty-cycled network joining with an appropriate capacitor size. Experimental results show that duty-cycled network joining is beneficial when there are more than one nodes that need to connect to the network. The network can be formed quicker than those with a $100 \%$ network join duty cycle. Appropriate sizing of the capacitor is also essential so that the energy provided to the node is enough to join the network from the first attempt. By using a $50 \%$ network join duty cycle and a $100 \mathrm{mF}$ capacitor, the AEH and VEH powered wireless sensor nodes formed a WSN in just one attempt successfully. Once the nodes have connected to the network, the energy harvesters can provide sufficient energy for perpetual operation of the wireless sensor nodes and recharge the capacitor simultaneously.

\section{REFERENCES}

[1] A. A. Kumar S, K. Øvsthus, and L. M. Kristensen, "An industrial perspective on wireless sensor networks - a survey of requirements, protocols, and challenges," IEEE Commun. Surveys Tuts., vol. 16, no. 3, pp. 1391-1412, 2014.

[2] R. Bogue, "Wireless sensors: A review of technologies, products and applications," Sens. Rev., vol. 30, no. 4, pp. 285-289, 2010.

[3] Y. K. Tan, and S. K. Panda, "Energy harvesting from hybrid indoor ambient light and thermal energy sources for enhanced performance of wireless sensor nodes," IEEE Trans. Ind. Electron., vol. 58, no. 9, pp. 4424-4435, 2011.

[4] Z. J. Chew, S. B. Tuddenham, and M. Zhu, "Airflow energy harvesting with high wind velocities for industrial applications," JPCS, vol. 773, no. 1, pp. 012091 (5 pp), 2016.

[5] A. C. Waterbury, and P. K. Wright, "Vibration energy harvesting to power condition monitoring sensors for industrial and manufacturing equipment," Proc. Inst. Mech. Eng. C J. Mech. Eng. Sci., vol. 227, no. 6, pp. 1187-1202, 2013.

[6] D. Rozgić, and D. Marković, "A miniaturized $0.78-\mathrm{mW} / \mathrm{cm}^{2}$ autonomous thermoelectric energy-harvesting platform for biomedical sensors," IEEE Trans. Biomed. Circuits Syst., vol. 11, no. 4, pp. 773-783, 2017.

[7] M. Raza, N. Aslam, H. Le-Minh, S. Hussain, Y. Cao, and N. M. Khan, "A critical analysis of research potential, challenges, and future directives in industrial wireless sensor networks," IEEE Commun. Surveys Tuts., vol. 20, no. 1, pp. 39-95, 2018.

[8] S. Sudevalayam, and P. Kulkarni, "Energy harvesting sensor nodes: Survey and implications," IEEE Commun. Surveys Tuts., vol. 13, no. 3, pp. 443-461, 2011.

[9] Z. J. Chew, T. Ruan, and M. Zhu, "Power management circuit for wireless sensor nodes powered by energy harvesting: On the synergy of harvester and load," IEEE Trans. Power Electron., pp. 1-1, 2018.

[10] Z. J. Chew, T. Ruan, and M. Zhu, "Strain energy harvesting powered wireless sensor system using adaptive and energy-aware interface for enhanced performance," IEEE Trans. Ind. Informat., vol. 13, no. 6, pp. 3006-3016, 2017.

[11] T. Watteyne, J. Weiss, L. Doherty, and J. Simon, "Industrial IEEE802.15.4e networks: Performance and trade-offs," in IEEE Int. Conf. Commun. (ICC), 2015, pp. 604-609.

[12] B. Martinez, X. Vilajosana, F. Chraim, I. Vilajosana, and K. S. J. Pister, "When scavengers meet industrial wireless," IEEE Trans. Ind. Electron., vol. 62, no. 5, pp. 2994-3003, 2015.

[13] N. Matthys, F. Yang, W. Daniels, S. Michiels, W. Joosen, D. Hughes, and T. Watteyne, " $\mu$ PnP-Mesh: The plug-and-play mesh network for the Internet of Things," in IEEE 2nd World Forum on Internet of Things (WFIoT), 2015, pp. 311-315.

[14] A. E. Xhafa, B. Campbell, and S. Hosur, "Towards a perpetual wireless sensor node," in IEEE Sensors, 2013, pp. 1-4.

[15] Y. Ma, Q. Ji, S. Chen, and G. Song, "An experimental study of ultra-low power wireless sensor-based autonomous energy harvesting system," $J$. Renew. Sustain. Energy, vol. 9, no. 5, pp. 054702, 2017.

[16] T. Watteyne, L. Doherty, J. Simon, and K. Pister, "Technical overview of SmartMesh IP," in 7th Int. Conf. Innov. Mob. Internet Serv. Ubiquitous Comput., 2013, pp. 547-551.

[17] K. S. J. Pister, and L. Doherty, "TSMP: Time synchronized mesh protocol," in Proc. LASTED Int. Symp. Distrib. Sens. Netw. (DSN), Orlando, Florida, USA, 2008 pp. 391-398.

[18] Z. J. Chew, T. Ruan, M. Zhu, M. Bafleur, and J. Dilhac, "A multifunctional device as both strain sensor and energy harvester for structural health monitoring," in IEEE Sensors, Orlando, FL, USA, 2016, pp. 1-3.

[19] Z. J. Chew, and M. Zhu, "Adaptive maximum power point finding using direct $V_{\mathrm{OC}} / 2$ tracking method with microwatt power consumption for energy harvesting," IEEE Trans. Power Electron., vol. 33, no. 9, pp. 8164$8173,2018$.

[20] T. Ruan, Z. J. Chew, and M. Zhu, "Energy-aware approaches for energy harvesting powered wireless sensor nodes," IEEE Sensors J., vol. 17, no. 7, pp. 2165-2173, 2017.

[21] Z. J. Chew, and M. Zhu, "Threshold voltage control to improve energy utilization efficiency of a power management circuit for energy harvesting applications," Proceedings, vol. 2, no. 13,pp. 1052 (5 pp), 2018.

[22] Analog Devices, "SmartMesh IP application notes," Datasheet., 2016. [Online]. Available: https://www.analog.com/media/en/technicaldocumentation/application-notes/smartmesh_ip_application_notes.pdf. Accessed on Mar. 12, 2019.

[23] Z. J. Chew, and M. Zhu, "Adaptive self-configurable rectifier for extended operating range of piezoelectric energy harvesting," IEEE Trans. Ind. Electron., pp. 1-1, 2019.

[24] J. Xu, Y. Dong, Z. Zhang, S. Li, S. He, and H. Li, "Full scale strain monitoring of a suspension bridge using high performance distributed fiber optic sensors," Meas. Sci. Technol., vol. 27, no. 12, pp. 124017 ( 11 pp), 2016.

[25] T. Ji, B. R. Ellis, and A. J. Bell, "Horizontal movements of frame structures induced by vertical loads," Proc. Inst. Civil Eng.-Struct. Build., vol. 156, no. 2, pp. 141-150, 2003.

[26] Ø. Andreassen, C. E. Wasberg, A. Helgeland, M. Tutkun, J. C. Kielland, B. A. P. Reif, Ø. Lundberg, and A. Skaugen, Studies of aerodynamically induced vibrations on the $P-3 C$ maritime surveillance aircraft and proposed vibration reducing measures, FFI-rapport 2013/00245, vol. 385301, Norwegian Defence Research Establishment (FFI), 2013. 